\title{
Defect Structure of Mg-Doped GaN Nanowires
}

\author{
Y. Wu ${ }^{*}$, J.P. Zhang ${ }^{*}$, G. S. Cheng ${ }^{* *}$, M. Moskovits ${ }^{* *}$, and J.S. Speck ${ }^{*}$ \\ *Materials Department, University of California, Santa Barbara, CA 93106 \\ **Chemistry Department, University of California, Santa Barbara, CA 93106
}

With a large direct energy gap, $\mathrm{GaN}$ is a promising candidate for blue and ultraviolet light emitters. Quasi one-dimensional GaN nanowires may find application in optoelectrical devices and are interesting for fundamental research. $p-n$ junctions have recently been developed in $\mathrm{GaN}$ nanowires by first growing n-type nanowires and then subsequently continuing the growth in an environment to realize p-type material (via Mg-doping)[1]. In this work we have thoroughly examined thin $\mathrm{Mg}$-doped $\mathrm{GaN}$ nanowires as well as the unintentionally-doped ones. It has been found that doped nanowires show a high density of stacking faults when the diameter is less than $22 \mathrm{~nm}$. It is interesting that these defective structures are rarely observed in the unintentionally-doped GaN nanowires or in nanowires with larger diameters.

Unintentionally-doped GaN nanowires were synthesized by a vapor-liquid-solid (VSL) process at $980^{\circ} \mathrm{C}$ in a tube furnace. p-type doping was realized by incorporating $\mathrm{Mg}$ in the In-Ga-N precursor liquid through the decomposition of $\mathrm{Mg}_{3} \mathrm{~N}_{2}$ placed in a separate crucible for the second growth of the GaN nanowires[1]. TEM samples of the two kinds of nanowires were supported by holy carbon films and examined with JEOL 2010HR and JEOL2000FX operated at $200 \mathrm{kV}$.

All of the 15 examined $\mathrm{Mg}$-doped GaN nanowires, of which the diameters are less than $22 \mathrm{~nm}$, showed defective structures, which are mainly the mixture of hexagonal (h-GaN) and cubic $\mathrm{GaN}$ (c-GaN) units due to the stacking faults. It has been found that in this range of width, the nanowires are mostly oriented with the close-packing planes, (0001) of h-GaN or (111) of c$\mathrm{GaN}$, parallel to or nearly to the growth direction. A typical example is shown in Fig.1, where the lower portion of the nanowire, about $40 \%$ of the total width, is defect-free h-GaN crystal, and in the defective region above, besides the simple stacking of $\mathrm{AB}$ and $\mathrm{ABC}$, includes more complicated stacking sequences which can be interpreted as the "building blocks" of polytypism. So far, the possible polytype structures identified with high resolution images, as well as electron diffraction patterns, are 9R(ABCBCACAB), 4H(ABCB) and $12 \mathrm{H}(\mathrm{ACBCBCBCBCBC})$. It was noticed that the $\mathrm{GaN}$ nanowires below $22 \mathrm{~nm}$ in diameter were mostly curved as shown in Fig.2(b), and the defective regions were domains. The dimension of the domains was about tens of nanometers along the nanowires, and the domains were separated by Schockley partial dislocations. In contrast, only 2 in 10 non-doped GaN nanowires observed in the same diameter range display defective structures, which implies that the formation of the defective structure should be attributed to the introduction of $\mathrm{Mg}$ or temperature during the synthesis. In the case of MBE-grown $\mathrm{GaN}, \mathrm{Mg}$ is known to enhance extended defect formation [2].

\section{References}

[1] Guosheng Cheng et al., Appl. Phys. Lett. (to be published).

[2] This research was supported by Department of Energy under contract number 8-48249025005. The work also made use of the MRL Center Facilities at University of California, at Santa Barbara. 


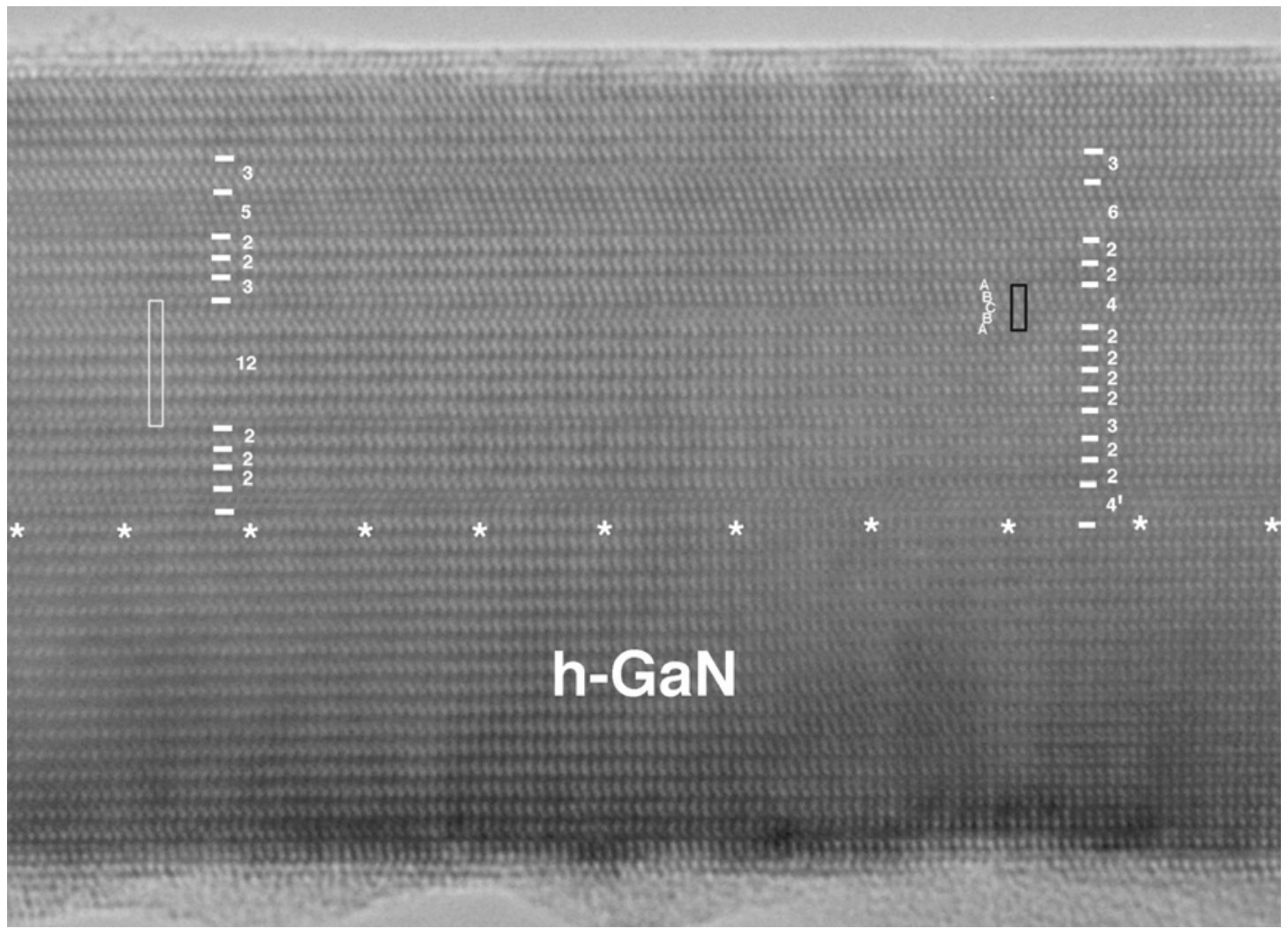

Fig. 1 High resolution image from a $\sim 22 \mathrm{~nm}$ diameter $\mathrm{GaN}$ nanowire. The row of stars separate the defective area (above) and the stacking-fault-free $\mathrm{h}-\mathrm{GaN}$ region (below). The numbers indicate the different stacking sequence: 2- $\mathrm{AB}, 3-\mathrm{ABC}, 4-\mathrm{ABCB}, 4$ '-ABAC, 5$\mathrm{ACBAC}, 6-\mathrm{ABACBA}$, and 12-ACBCBCBCBCBC. The white and black rectangles mark the unit cells of $12 \mathrm{H}$ and $4 \mathrm{H}$, respectively.
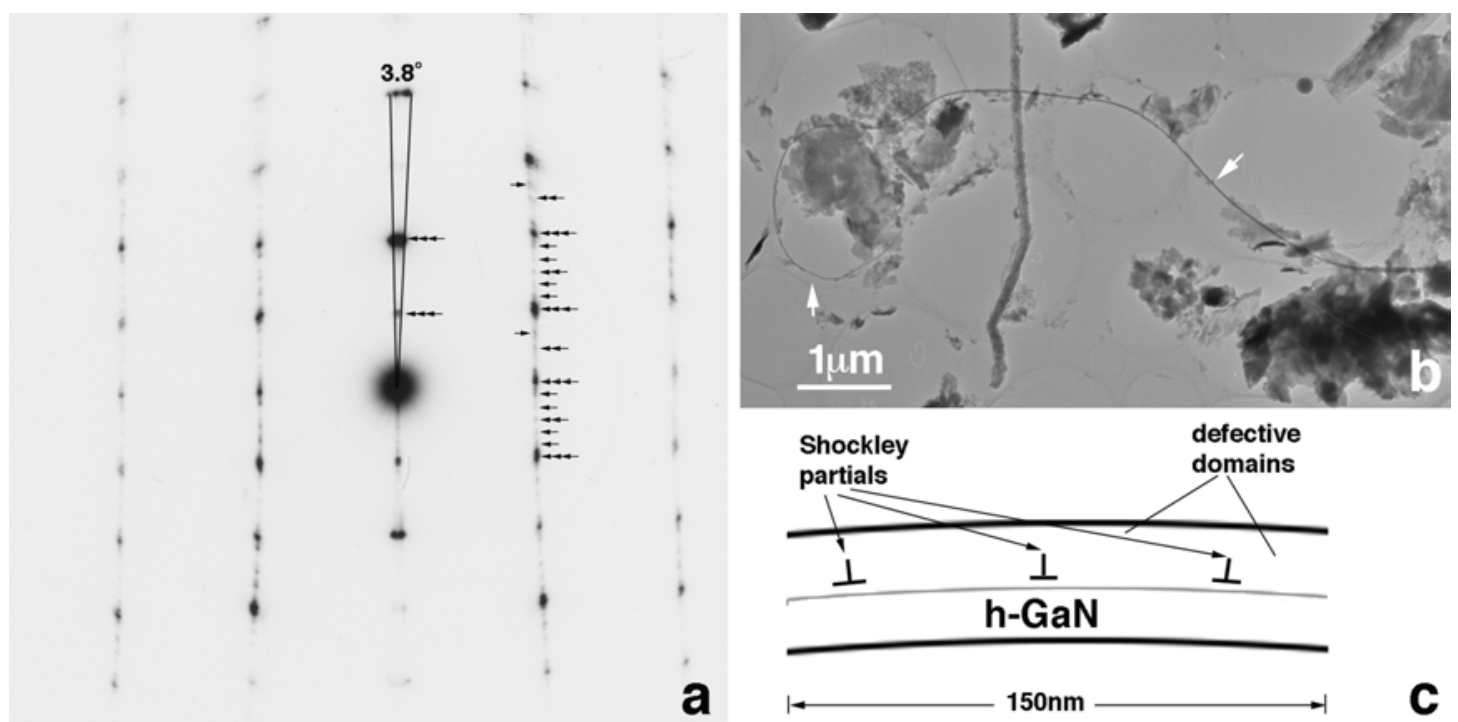

Fig. 2 (a) Electron diffraction pattern taken from the nanowire shown in Fig.1, indicating the existence of $12 \mathrm{H}$ (single-arrows), $4 \mathrm{H}$ (double-arrows), 2H(triple-arrows) and cubic (singlearrow on left). The helix angle of $3.8^{\circ}$ resulted from the curvature of the GaN nanowire, as illustrated in (b). A diagram of the defect structure in the nanowires in (c). 